\title{
The Proneural Basic Helix-Loop-Helix Gene Ascl1a Is Required for Retina Regeneration
}

\author{
Blake V. Fausett, Jessica D. Gumerson, and Daniel Goldman \\ Molecular and Behavioral Neuroscience Institute and Department of Biological Chemistry, University of Michigan, Ann Arbor, Michigan 48109
}

\begin{abstract}
Unlike mammals, teleost fish can regenerate an injured retina, restoring lost visual function. Little is known of the molecular events that underlie retina regeneration. We previously found that in zebrafish, retinal injury stimulates Müller glia to generate multipotent $\alpha 1$ tubulin $(\alpha 1 T)$ and pax6-expressing progenitors for retinal repair. Here, we report the identification of a critical E-box in the $\alpha 1 T$ promoter that mediates transactivation by achaete-scute complex-like la (asclla) during retina regeneration. More importantly, we show that asclla is essential for retina regeneration. Within $4 \mathrm{~h}$ after retinal injury, ascl1a is induced in Müller glia. Knockdown of asclla blocks the induction of $\alpha 1 T$ and pax 6 as well as Müller glial proliferation, consequently preventing the generation of retinal progenitors and their differentiated progeny. These data suggest asclla is required to convert quiescent Müller glia into actively dividing retinal progenitors, and that asclla is a key regulator in initiating retina regeneration.
\end{abstract}

Key words: ascl1a; Müller glia; stem cells; regeneration; retina; zebrafish

\section{Introduction}

Recent studies suggest that after retinal injury, Müller glia can dedifferentiate and function as retinal stem cells in mammals, birds, and fish (Fischer and Reh, 2001; Ooto et al., 2004; Fausett and Goldman, 2006; Bernardos et al., 2007; Fimbel et al., 2007). However, unlike mammals and birds, where the capacity of Müller glia to regenerate new neurons is extremely limited (Fischer and Reh, 2001; Ooto et al., 2004), teleost fish mount a robust regenerative response that not only regenerates all damaged retinal neurons (Vihtelic and Hyde, 2000; Fausett and Goldman, 2006; Bernardos et al., 2007; Fimbel et al., 2007), but also results in restoration of visual function (Mensinger and Powers, 2007). Therefore, teleost fish, such as zebrafish, provide an ideal model system for identifying the molecular mechanisms underlying a robust regenerative response after retinal injury and may suggest new strategies for repairing the damaged mammalian retina. Toward this goal, global changes in gene expression have been documented in the injured zebrafish retina (Cameron et al., 2005; Kassen et al., 2007), and these studies provide a list of candidate genes that may participate in specific events associated with regeneration. Although the temporal and spatial expression pattern of some of these candidate genes suggests a role in various stages

Received Oct. 25, 2007; revised Dec. 3, 2007; accepted Dec. 4, 2007.

This work was supported by a research grant from the National Eye Institute and by funds from the University of Michigan Endowment for the Basic Sciences (D.G.). B.V.F. was supported in part by a National Institutes of Health Vision Research Training grant. We thank members of the Goldman laboratory and Professors Ben Novitch and Dave Turner for comments and suggestions on this work. We also thank Eric Weinberg, Matthias Hammerschmidt, Bruce Appel, Julian Lewis, Michael Lardelli, and Audrey Seasholtz for providing CDNA clones. We thank Richard Griggs for generating custom electroporation electrodes.

Correspondence should be addressed to Daniel Goldman, University of Michigan, 5045 BSRB, 109 Zina Pitcher Place, Ann Arbor, Ml 48109-2200. E-mail: neuroman@umich.edu.

DOI:10.1523/JNEUROSCI.4853-07.2008

Copyright $\odot 2008$ Society for Neuroscience $\quad$ 0270-6474/08/281109-09\$15.00/0 of regeneration (Yurco and Cameron, 2007), they do not inform us of the significance of these gene expression patterns.

Another approach for uncovering mechanisms of regeneration is to use promoters of regeneration-associated genes as reporters for transcription factors and other signaling molecules that mediate gene induction during retina regeneration. We have taken this approach using the $\alpha 1$-tubulin $(\alpha 1 T)$ promoter as a reporter for the regenerative response. In this case, transgenic zebrafish were created that harbor the $\alpha 1 T$ promoter driving enhanced green fluorescent protein (EGFP) expression $(\alpha 1 T: G F P)$ (Goldman et al., 2001). Using these fish, we recently showed that transgene-expressing Müller glia are a source of multipotent progenitors that contribute newborn cells toward retina regeneration in zebrafish (Fausett and Goldman, 2006). Here, we report the identification of an E-box within the $\alpha 1 T$ promoter that is required for its induction after retinal injury and provide evidence that the proneural basic helix-loop-helix (bHLH) transcription factor asclla regulates the $\alpha 1 T$ promoter via this E-box. Most importantly, we found that asclla is induced within $4 \mathrm{~h}$ after retinal injury, and knockdown of asclla prevents induction of regeneration-associated genes, such as $\alpha 1 T$ and pax6, and also inhibits Müller glia proliferation in response to injury. These data suggest asclla is a key transcription factor that is involved in initiating regeneration in the injured retina.

\section{Materials and Methods}

Animals. The animals used in this study were treated in accordance with the guidelines of the University Committee on Use and Care of Animals at the University of Michigan. Fish were obtained from our breeding

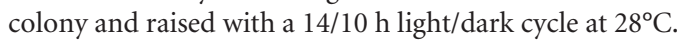

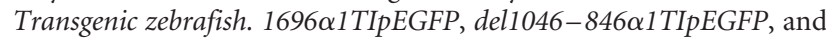
$1016 \alpha 1$ TIPEGFP transgenic fish have been described previously (Goldman and Ding, 2001; Goldman et al., 2001; Fausett and Goldman, 2006). -907 11 TIPEGFP and TG-954CA 11 TIPEGFP constructs were resuspended in injection buffer, and single-cell zebrafish embryos were in- 
a

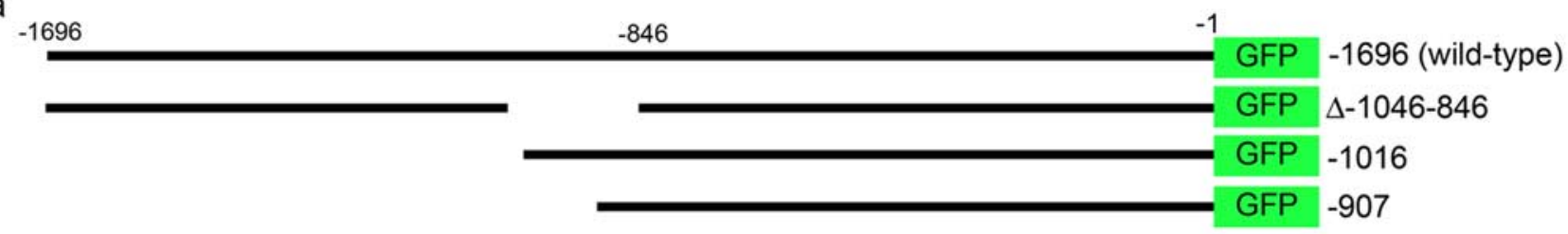

b

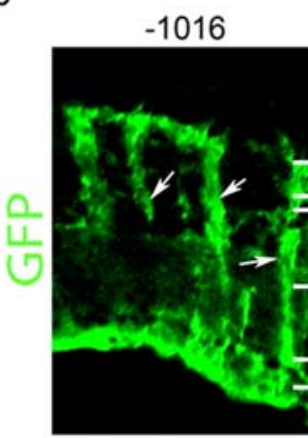

$\Delta-1046-846$ L1

$\Delta-1046-846$ L3
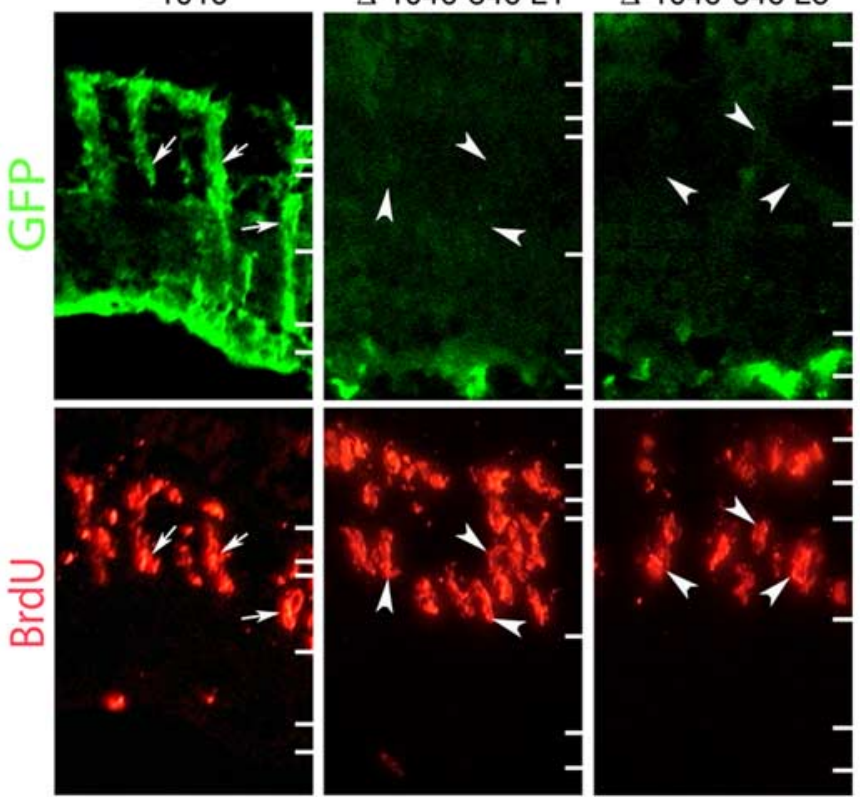

-907 L1

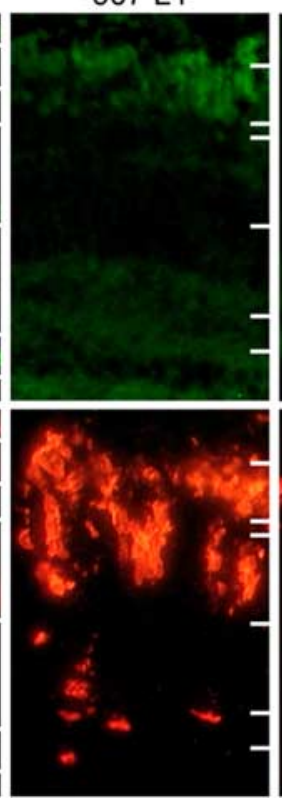

-907 L2

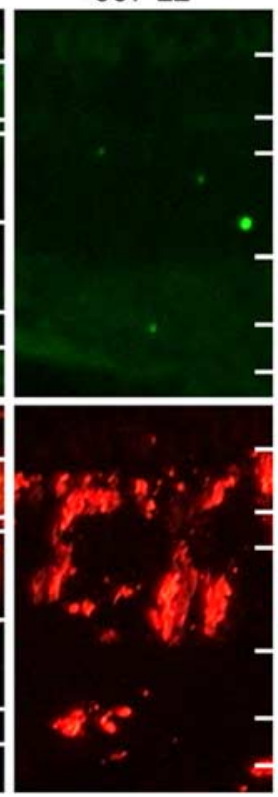

-907 L3

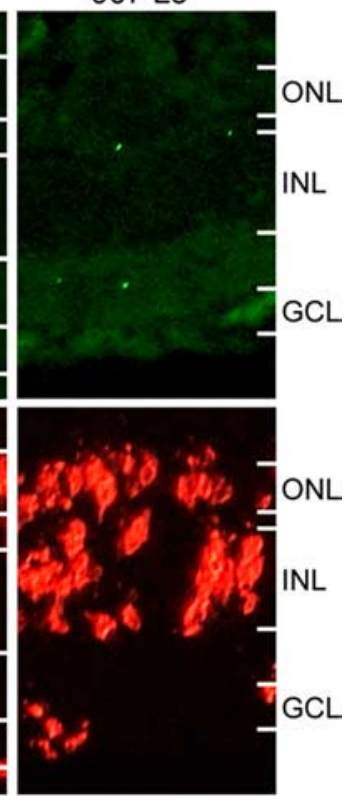

Figure 1. A 109 bp region of the $\alpha 1 T$ promoter is required for transgene expression in dedifferentiating Müller glia. $a$, Schematic representation of $\alpha 1 T$ promoter constructs. The bars represent promoter sequence, and the numbers indicate relative position from the start codon. - 1696 is the wild-type promoter described previously (Hieber et al., 1998; Goldman et al., 2001). $\Delta$-1046 - 846 has been described previously (Goldman and Ding, 2000). The -1016 promoter directs transgene expression in Müller glia (Fausett and Goldman, 2006). The -907 promoter lacks 789 bp of upstream sequence. $\boldsymbol{b}$, Transgenic fish received retinal injuries on day 0 and were given a $4 \mathrm{~h}$ pulse of BrdU at $4 \mathrm{dpi}$. Transgenic fish, which carry the required DNA element, express GFP in BrdU-labeled Müller glia (-1016, arrows), whereas transgenic fish lacking the element do not $(\Delta-1046-846$ and -907$)$. Two independent lines of $\Delta-1046-846$ and three independent lines of -907 transgenic fish all display a lack of GFP expression in BrdU-labeled cells (arrowheads in $\Delta$-1046 - 846). The images for -1016 and $\Delta-1046-846$ are from the same sections. Because the -907 transgenic fish display very weak GFP expression in general, we used serial sections to obtain the -907 images. GCL, Ganglion cell layer.

jected, raised to adulthood, and screened for transgenic progeny as described previously (Goldman and Ding, 2001; Goldman et al., 2001).

Optic nerve lesions, eye lesions, and Morpholino-mediated gene knockdown. Fish were anesthetized in $0.02 \%$ tricaine methane sulfonate (Sigma, St. Louis, MO) before surgery. Optic nerve crushes were performed as described previously (Hieber et al., 1998; Senut et al., 2004; Fausett and Goldman, 2006). Eye lesions were performed as described previously (Senut et al., 2004; Fausett and Goldman, 2006). Briefly, fish were anesthetized and under microscopic visualization, the right eye was gently pulled from its socket and stabbed four times (once in each quadrant) through the sclera with a 30 gauge needle. To deliver morpholinos to the injured retina, a 30 gauge needle was attached to a Hamilton syringe (Hamilton, Reno, NV) containing $1 \mathrm{~mm}$ morpholino (Gene Tools, Philomath, OR). Approximately $0.5 \mu \mathrm{l}$ was injected into the vitreous after inserting the needle to the length of the bevel. We used the following lissamine-labeled morpholinos: control morpholino oligonucleotide (MO) 5'CCTCTTACCTCAGTTACAATTTATA-3'; asclla

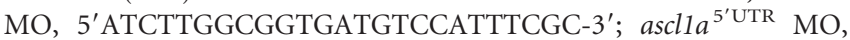
5'AAGGAGTGAGTCAAAGCACTAAAGT-3' [the latter two MOs have been described previously as ash1a MOs (Cau and Wilson, 2003)]. Custom tweezer-like electrodes (stainless steel, $4 \mathrm{~mm}$ wide, $25 \mathrm{~mm}$ length, $0.2 \mathrm{~mm}$ thickness) were then placed across the head of the fish with the cathode on the left eye and the anode on the right eye. An ECM 830 Electro Square Porator (BTX, San Diego, CA) was used to deliver five consecutive $50 \mathrm{~ms}$ pulses at $70 \mathrm{~V}$ with a $950 \mathrm{~ms}$ interval between pulses. The uninjected eye served as a negative control. One observer assigned letters to control and ascl1a MOs. A second observer then electroporated these MOs into fish and assigned the fish with numbers. This way, both observers could score MO-treated cells for GFP expression without any bias. For cell counts, morpholino-treated cells were scored for either GFP or bromodeoxyuridine (BrdU) labeling. The total number of morpholino-labeled cells that were labeled with GFP or BrdU was tallied for each retina to determine the percentage of MO-treated cells, which were also labeled with GFP or BrdU. The percentage of double-labeled cells for each group was calculated by taking the average from three retinas. A student's $t$ test, assuming equal variance, was used to calculate $p$ values.

Bromodeoxyuridine labeling. To identify dividing cells, fish were either given a single injection of BrdU as described previously (Fausett and Goldman, 2006) or housed in $10 \mathrm{~mm}$ BrdU for $24 \mathrm{~h}$ [from 24-48 or $36-60 \mathrm{~h}$ postinjury (hpi)]. Fish were transferred to tanks with fresh water and killed at various times after BrdU administration to harvest the retinas.

Tissue preparation, immunohistochemistry, and in situ hybridization. Fish were given an overdose of tricaine methane sulfonate, and the eyes from adult fish were dissected, enucleated, fixed, and sectioned as described previously (Senut et al., 2004; Fausett and Goldman, 2006). Immunohistochemistry was performed as described previously (Senut et al., 2004; Fausett and Goldman, 2006). The following primary antibodies were used: rat anti-BrdU (dividing cell marker; 1:250; Harlan, Sera-Lab, Sussex, UK); rabbit anti-GFP (1:1000; Invitrogen, Eugene, OR); and mouse anti-glutamine synthetase (GS) (glial marker; 1:500; Millipore, Temecula, CA). In situ hybridizations (ISHs) were performed with digoxigenin-labeled cRNA probes as described previously (Barthel and Raymond, 2000). Asclla was a gift from Eric Weinberg (University of Pennsylvania, Philadelphia, PA); Pax6 cRNA was prepared from a full- 


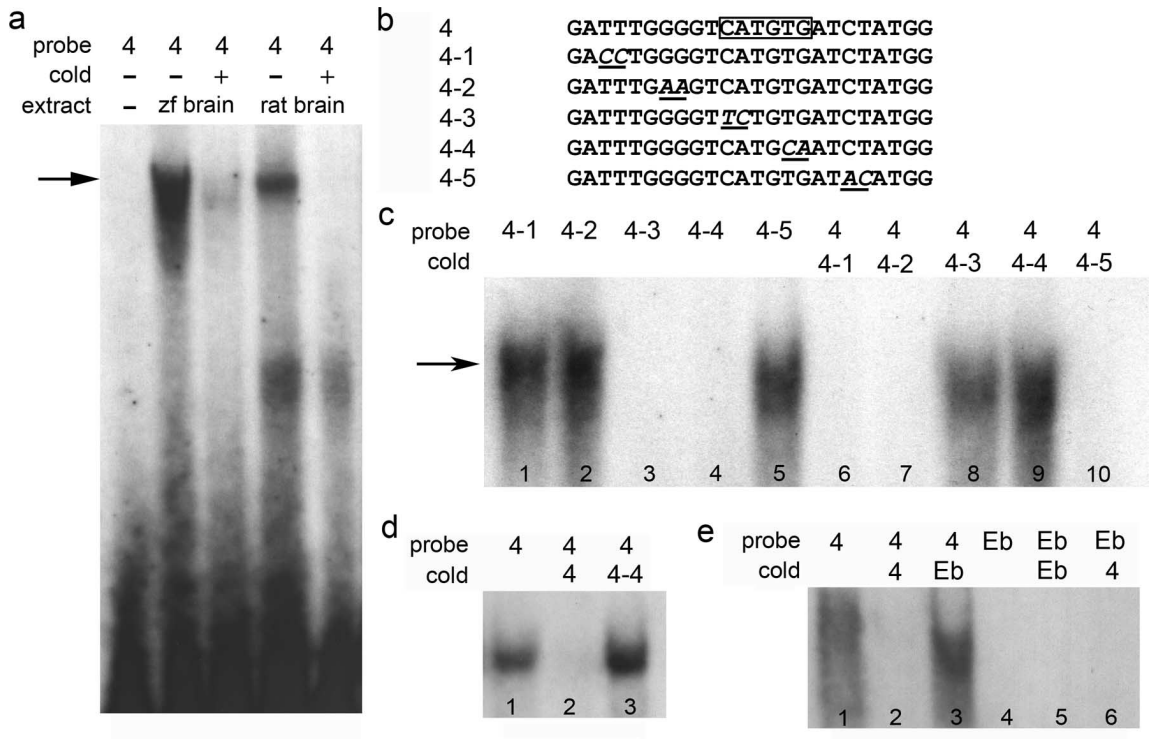

Figure 2. An E-box within the $109 \mathrm{bp} \mathrm{region}$ that is required for transgene expression in Müller glia binds nuclear extracts from zebrafish and rat brain and zebrafish retina. $\boldsymbol{a}$, Electrophoretic mobility shift assay using a probe from the $109 \mathrm{bp}$ region binds nuclear extracts from zebrafish and rat brain. The arrow indicates specific binding. Cold indicates where 50-fold molar excess unlabeled probe was added as competition. Extract indicates whether zebrafish (zf) or rat brain extracts were added. $\boldsymbol{b}$, Nucleotide sequence of the probes used for electrophoretic mobility shift assay. The E-box is outlined in probe 4 with a box. Mutations are indicated by italicized and underlined text. c, Mutations to the E-box (lanes 3 and 4) render the probe unable to bind nuclear extracts from zebrafish brain, whereas mutations to non-E-box nucleotides do not affect binding (lanes 1, 2, and 5). Probes correspond to those shown in $\boldsymbol{b}$. Unlabeled mutant probes compete with wild-type probe binding when the E-box is intact (lanes 6,7 , and 10), but not when the E-box is mutated (lanes 8 and 9 ), even at 50 -fold molar excess. $\boldsymbol{d}$, Nuclear extracts from zebrafish retina bind specifically to the E-box. $\boldsymbol{e}$, An E-box probe from a different region of the promoter (Eb) is unable to compete with the E-box from probe 4 (lane 3) and does not bind to zebrafish brain nuclear extracts (lane 4).

length Pax6a cDNA clone (Open Biosystems, Huntsville, AL); $\alpha 1$ T probe was described previously (Hieber et al., 1998); notch1b and notch3 were gifts from Michael Lardelli (University of Adelaide, Adelaide, Australia); deltaA, deltaD, and olig2 were gifts from Bruce Appel (Vanderbilt University, Nashville, TN); and deltaB was a gift from Julian Lewis (UCL, London, UK). For timeline expression analysis (supplemental Table 1, available at www.jneurosci.org as supplemental material), notch 3 hybridization was done individually and in combination with notch $1 b$; similar results were obtained in each case. Delta in situ hybridization was done with deltaA individually and in combination with deltaB and deltaD; similar results were obtained in each case. Negative results were repeated twice for the 24 and 48 hpi time points except for olig2.

Imaging. Slides were examined using a Zeiss (Oberkochen, Germany) Axiophot or Olympus (Tokyo, Japan) Fluoview FV1000 laser scanning confocal microscope. Images were captured using a digital camera adapted onto the Axiophot microscope or Olympus confocal microscope. Images were processed and annotated with Adobe Photoshop CS (Adobe Systems, San Jose, CA).

Vectors. $-907 \alpha 1$ TIPEGFP expression vector contains $907 \mathrm{bp}$ of $5^{\prime}$ flanking $\alpha 1$-tubulin DNA, exon 1, and the first intron fused in frame to the GFP sequence. This promoter fragment is similar to the full-length - 1696 21TIPEGFP expression vector from previous work (Goldman and Ding, 2000; Goldman et al., 2001; Senut et al., 2004), except that it is lacking $789 \mathrm{bp}$ from the $5^{\prime}$ end. A 2 bp mutation (TG-CA) was introduced into the full-length $\alpha 1 T$ promoter by amplifying a PCR product containing the $2 \mathrm{bp}$ E-box mutation and cloning it into the $\alpha 1$ TIPEGFP vector. The TG-954CA $\alpha 1$ TIPEGFP construct is identical to the -1696 expression vector except for the TG:CA mutation at position -954. Luciferase reporter vectors are based on the $p X P 2$ construct (Nordeen, 1998 ). The $\alpha 1$ TpXP2 reporter (wild-type) contains 1696 bp of 5 ' flanking $\alpha 1 T$ sequence and its first exon cloned in frame and upstream of the luciferase coding sequence. The TG-954CA $\alpha 1 T-p X P 2$ vector is identical to the wild-type vector except for a 2 bp substitution at position 954 (TG-CA). The E-box-pXP2 vector contains three copies of the $\alpha 1 T$ E-box at position 954 (5'CATGTG $\left.3^{\prime}\right)$ upstream of a minimal $\beta$-globin promoter in $p X P 2$. The $m E$ box- $p X P 2$ vector is identical to the $E-b o x-p X P 2$ vector, except each E-box has a 2 bp mutation (CATGTG to CATGCA). The coding sequence of asclla was cloned into pCS2+ to create $p C S 2+$ ascl1a. $p C S 2+\beta$-globin was a gift from Audrey Seasholtz (University of Michigan, Ann Arbor, MI).

Transactivation assays. Human embryonic kidney 293T (HEK293T) cells were plated in 24-well plates $24 \mathrm{~h}$ before transfection. Cells were transfected via calcium phosphate DNA precipitation. Luciferase assays were performed in duplicate for each sample, and values were normalized to $\beta$-globin expression.

Nuclear extracts and electrophoretic mobility shift assays. Nuclear extracts were isolated from zebrafish brain, retina, and HEK293T transfected cells using standard protocols. Oligonucleotide probes labeled with ${ }^{32} \mathrm{P}$-dCTP (ICN, San Diego, CA) were incubated with 5-10 $\mu$ g of nuclear extracts prepared from zebrafish brain or retina, rat brain, or with in vitro synthesized proteins using TNT Coupled Transcription/ Translation system (Promega, Madison, WI). Cold oligonucleotides were incubated at $>50$ fold excess to test binding specificity. The protein:DNA mix was then run on a nondenaturing polyacrylamide gel, and DNA migration was visualized by exposing the gel to Kodak (Rochester, NY) imaging film.

\section{Results}

\section{An E-box is required for $\alpha 1 T$ promoter activation in proliferating Müller glia}

after retinal injury

The $-1696 \alpha 1$ T:GFP and $-1016 \alpha 1$ T:GFP transgenes are induced in proliferating Müller glia after retinal injury (Senut et al., 2004; Fausett and Goldman, 2006). Identification of DNA elements mediating transgene induction may provide insight into the mechanism by which Müller glia respond to injury-induced signals and produce a dividing population of retinal stem cells. We hypothesized that these elements would lead us to transcription factors that regulate retina regeneration. Therefore, transgenic fish harboring different $\alpha 1 T$ promoter deletions driving GFP expression were screened for transgene expression in proliferating Müller glia after retinal injury (Fig. 1). This screen confirmed the $-1016 \alpha 1 T$ :GFP transgene was induced after retinal injury and identified $\Delta$-1046-846 11 :GFP and $-907 \alpha 1 T$ :GFP as transgenes that no longer responded to retinal injury by inducing GFP expression in proliferating Müller glia (Fig. 1). This lack of expression is not attributable to promoter inactivation, because these promoters can drive transgene expression during development (Goldman and Ding, 2000; our unpublished observations). These results suggest that a DNA element located between nucleotides -1016 and -907 of the $\alpha 1 T$ promoter is required for transgene expression in proliferating Müller glia.

To further narrow-in on this element, we searched for potential transcription factor binding sites within this $109 \mathrm{bp}$ region by performing gel electrophoretic mobility shift assays. Radiolabeled oligonucleotide probes spanning different parts of the 109 bp sequence were incubated with nuclear protein extracts prepared from zebrafish or rat brain (Fig. 2a) or zebrafish retina (Fig. $2 d$ ) and resolved on native polyacrylamide gels. These experiments revealed specific binding of nuclear proteins to probe 4 
(Fig. 2a, arrow), which contains a single E-box sequence CATGTG (Fig. 2b). Two base pair mutations within the E-box (Fig. $2 b$, probes $4-3,4-4$ ) disrupted protein binding (Fig. $2 c$, lanes 3 , 4), whereas mutations in surrounding nucleotides (Fig. 2b, probes $4-1,4-2,4-5$ ) had no effect on binding (Fig. $2 c$, lanes 1 , $2,5)$. In addition, E-box mutations prevented unlabeled probes from competing for binding with wild-type radiolabeled probes even at 50 -fold molar excess (Fig. $2 c$, lanes 8,9 ). Although nuclear extracts prepared from brain or retina bound probe 4 (Fig. 2), those prepared from rat liver, fish gill, fish muscle, and fish kidney did not (data not shown). To further test the specificity of this binding, we used a probe (Eb), which contains an E-box (CAGATG), that is not required for transgene expression after retinal injury but is required for transgene induction in retinal ganglion cells after optic nerve crush (Senut et al., 2004). Unlike the probe harboring the E-box at position -954, probe Eb did not bind nuclear proteins from zebrafish brain (Fig. $2 e$, lanes $4-6$ ). These results suggest that the binding we observe is specific to the E-box at position -954 .

Might this E-box mediate transgene induction in Muller glia after retinal injury? To investigate this question, we placed the 2 bp mutation from probe $4-4$ (Fig. $2 b$ ) in the full-length $-1696 \alpha 1 T$ promoter to create TG-954CA $\alpha 1 T$ :GFP transgenic zebrafish (Fig. 3a). Three independent lines of transgenic fish were identified and examined for transgene expression during development. The TG-954CA promoter directs transgene expression to the brain, spinal cord, and retina during development, which is similar to the wild-type promoter (supplemental Fig. 1, available at www.jneurosci.org as supplemental material). We reported previously that mature fish harboring the $-1696 \alpha 1 T$ : GFP or $-1016 \alpha 1 T$ :GFP transgene express GFP in retinal stem cells located at the circumferential germinal zone (CGZ) (Goldman et al., 2001; Fausett and Goldman, 2006), where new retinal cells are continually added as the eye grows. Interestingly, TG954CA $\alpha 1$ T:GFP fish do not express the transgene in the CGZ (supplemental Fig. 2, available at www.jneurosci.org as supplemental material), suggesting the E-box is required for transgene expression in retinal stem cells. Consistent with the idea that this particular E-box is essential for $\alpha 1 T$ expression in adult retinal stem cells is our observation that after retinal lesion, TG-954CA $\alpha 1$ :GFP fish do not express GFP in proliferating Müller glia (Fig. 3b). Occasionally we noticed GFP ${ }^{+}$cells in the ganglion cell layer after retinal lesion (Fig. $3 b$, arrows). These appeared to be axotomized retinal ganglion cells, which are known to induce expression from the $-1696 \alpha 1$ : GFP transgene (Goldman and Ding, 2000; Goldman et al., 2001; Senut et al., 2004). We confirmed this in TG-954CA 11 : GFP fish by observing robust GFP expression in retinal ganglion cells after optic nerve crush (supplemental Fig. 3, available at www.jneurosci.org as supplemental material). Therefore, the identified E-box is specifically necessary for transgene expression in adult retinal stem cells residing in either the retinal periphery (CGZ) or in the central retina after injury (proliferating Müller glia).

\section{Ascl1 $a$ is rapidly induced in Müller glia after retinal injury}

The results described above suggest basic helix-loop-helix transcription factors may be crucial for retina regeneration. One such protein, asclla, attracted our attention because of the following: (1) the chick homolog of asclla is expressed in Müller gliaderived progenitors in the injured chick retina (Fischer and Reh, 2001); (2) asclla is induced in Müller glia during regeneration of the zebrafish retina (Yurco and Cameron, 2007); and (3) the mouse homolog of asclla is expressed in retinal (Jasoni and Reh, a

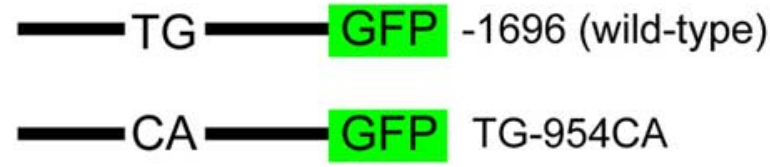

b
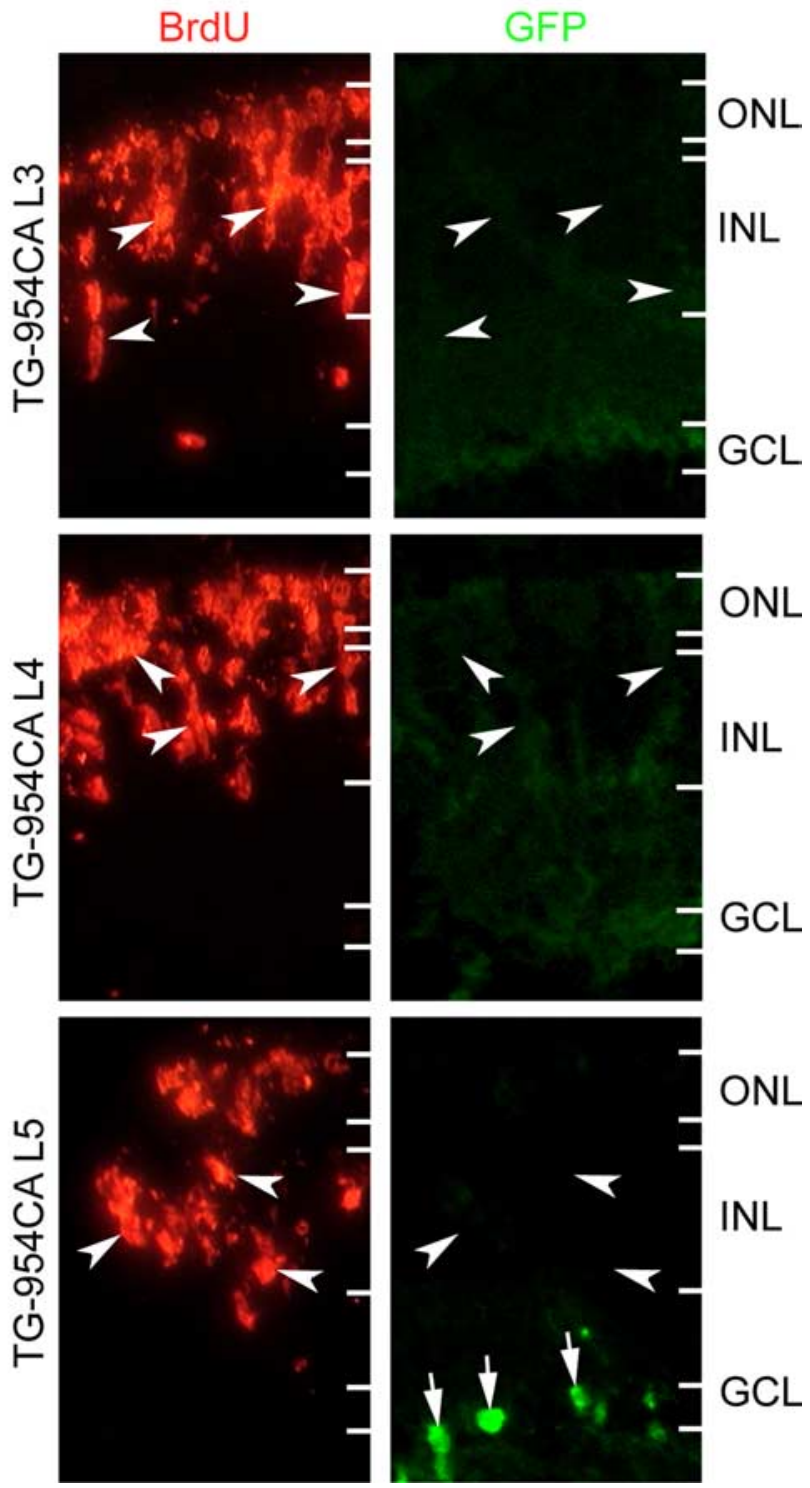

Figure 3. Mutation of $-954 \mathrm{E}$-box in the wild-type $-1696 \alpha 1 T$ promoter prevents transgene induction in proliferating Müller glia after retina injury. $\boldsymbol{a}$, Schematic representation of $\alpha 1 T$ promoter:GFP constructs. The wild-type $-1696 \alpha 1$ T:GFP transgene (-1696) and the $2 \mathrm{bp}$ mutation created in the E-box at position - 954 in the -1696 construct (TG-954CA) are indicated. $\boldsymbol{b}$, Three independent lines of transgenic fish received retinal injuries on day 0 and were given a $4 \mathrm{~h}$ pulse of BrdU at $4 \mathrm{dpi}$. Note that none of the lines harboring the $2 \mathrm{bp}$ mutation induced transgene GFP expression in BrdU-labeled Müller glia (arrowheads). See Figure 1 for comparison with the wild-type $-1696 \alpha 1$ T:GFP transgene response. The arrows in the lowest right GFP panel show transgene induction in retinal ganglion cells, the axons of which were presumably damaged when we injured the retina in this fish.

1996) and neural (Torii et al., 1999; Yun et al., 2002) progenitors. To confirm asclla was induced in proliferating Müller glia, we examined injured retinas from -1016 1T:GFP fish for ascl1a expression. We observed that asclla expressing cells correspond 

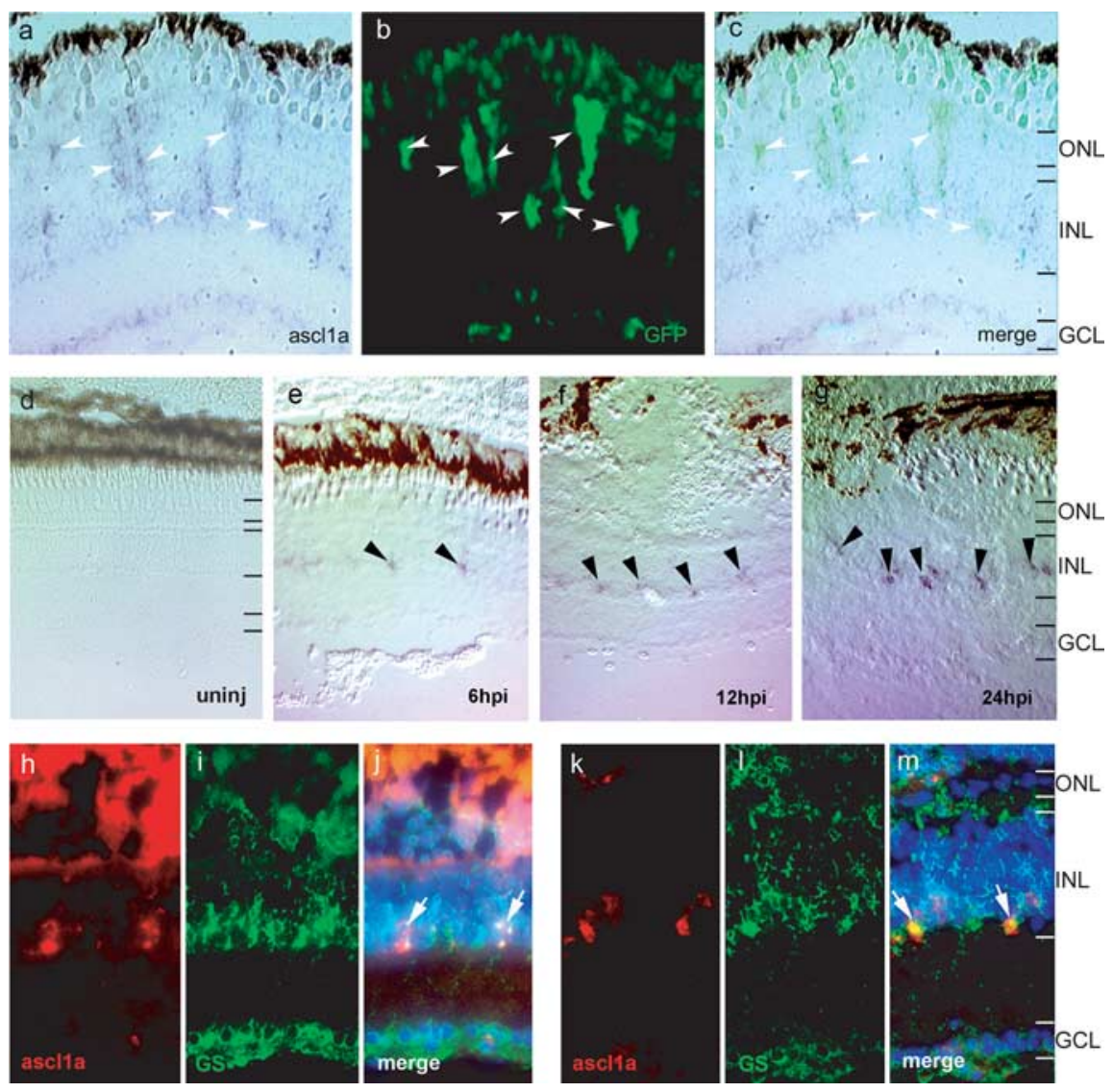

Figure 4. Ascl1a is induced in proliferating Müller glia after retinal injury. $\boldsymbol{a}-\boldsymbol{c}$, Ascl1a expression is detected by in situ hybridization ( $\boldsymbol{a}$ ) in GFP ${ }^{+}$Müller glia (b) at $4 \mathrm{dpi}$ (arrowheads). c shows the merge image of $\boldsymbol{a}$ and $\boldsymbol{b}$. $\boldsymbol{d}$ - $\boldsymbol{g}$, In situ hybridization for ascl1a from 6-24 hpi. Ascl1a is not expressed in the uninjured retina (d). Ascl1a is induced in cells of the INL at $6 \mathrm{hpi}(\boldsymbol{e}$, arrowheads). Ascl1a expression gradually increases and is easily detected at $24 \mathrm{hpi}$ ( $\boldsymbol{g}$, arrowheads). $\boldsymbol{h}-\boldsymbol{m}$, Ascl1a in situ hybridization (red, $\boldsymbol{h}, \boldsymbol{k}$ ) and glutamine synthetase immunostaining (green, $\boldsymbol{i}, \boldsymbol{I}$ ) show asclla is induced in Müller glia at $6 \mathrm{hpi}$ (arrows in merge, $\boldsymbol{j}$ ) and 24 hpi (arrows in merge, $\boldsymbol{m}$ ). DAPI nuclear staining is shown in the merged panels. GCL, Ganglion cell layer.

difficult to detect its induction until ectopic expression appeared in the inner and outer nuclear layers (ONLs) at $96 \mathrm{hpi}$. This result is consistent with the observation that not all GFP ${ }^{+}$Müller glia induce pax6 at 4 dpi (Fausett and Goldman, 2006), suggesting pax6 induction in Müller glia occurs after GFP induction. Although low levels of pax6 protein have been reported in Müller glia (Bernardos et al., 2007), we did not detect pax6 induction in transgene expressing Müller glia at 6 or 24 hpi (supplemental Fig. 4, available at www. jneurosci.org as supplemental material).

\section{Ascl1 a regulates $\alpha 1 T$ expression}

Because injury-induced expression of the $-1696 \alpha 1$ T:GFP transgene requires an E-box at position -954 and E-boxes bind basic helix-loop-helix transcription factors like asclla, we suspected asclla would regulate $\alpha 1 T$ promoter activity via its -954 E-box. Consistent with this idea, we found that asclla transactivated a minimal $\beta$-globin promoter harboring three copies of the -954 E-box (CATGTG) but did not transactivate the $\beta$-globin promoter with three copies of a mutant E-box (CATGCA) (Fig. 5a). In addition, mutation of this E-box in the context of the $-1696 \alpha 1 T$ promoter also blocked transactivation by ascl1a (Fig. 5b), although transactivation was less dramatic, possibly because of high basal expression caused by endogenous transcriptional activators, which act at sites other than the E-box. We were unsuccessful in demonstrating a direct interac-

precisely to $\mathrm{GFP}^{+}$Müller glia at $4 \mathrm{~d}$ postinjury (dpi) (Fig. $4 a-c$ ), which we previously identified as proliferating Müller gliaderived retinal progenitors (Fausett and Goldman, 2006).

To determine whether asclla expression precedes Müller glia proliferation and $\alpha 1 T$ induction, which begin at $\sim 24 \mathrm{hpi}$ (Fausett and Goldman, 2006), we examined retinas at 6, 12, 18, and 24 hpi for asclla expression by ISH. We detected a low level of expression at 6,12 , and $18 \mathrm{hpi}$ and stronger expression at $24 \mathrm{hpi}(6,12$, and 24 hpi shown in Fig. $4 d-g$ ). Colocalization of asclla and glutamine synthetase at these early time points confirmed ascllaexpressing cells are Müller glia (Fig. $4 h-m$ ). These results indicate that asclla is induced in Müller glia at least $18 \mathrm{~h}$ before they enter the cell cycle and begin expressing the $\alpha 1 T$ transgene.

To put asclia and $\alpha 1 T$ gene induction in the context of other genes induced in the injured retina, which are known to be involved in retina development and induced during retina regeneration, we compared ascl $1 a$ and $\alpha 1 T$ expression with pax6, delta, notch, and olig2 at various times after retinal injury using ISH. The results of this analysis are summarized in supplemental Table 1 (available at www.jneurosci.org as supplemental material) and show that asclla induction is the earliest indication of Müller glia dedifferentiation after retinal injury. Asclla was first detectable at $4 \mathrm{hpi}$, increasing at $24 \mathrm{hpi}$ and reaching peak levels by $48 \mathrm{hpi}$. In contrast endogenous $\alpha 1$ T expression increases at $24 \mathrm{hpi}$, whereas delta, notch, and olig 2 are induced between 48 and 96 hpi. Because pax6 is expressed by amacrine cells (Hitchcock et al., 1996), it was tion between asclla and the -954 E-box using gel electrophoretic mobility shift assays (our unpublished observations), which may suggest that asclla activation of the $\alpha 1 T$ promoter is indirect or requires the presence of additional DNA-binding and/or ascllainteracting proteins.

Despite the lack of evidence that asclla can bind the -954 E-box, the very early induction of asclla after retinal injury and its ability to regulate $\alpha 1 T$ promoter activity suggest it may regulate $\alpha 1 \mathrm{~T}$ transcription in vivo. To test this hypothesis, we suppressed asclla expression in Müller glia after retinal injury. To do this, we electroporated injured adult retinas with lissaminelabeled antisense MOs that have been demonstrated previously to knockdown asclla expression when injected into single-cell zebrafish embryos (Cau and Wilson, 2003). We compared the effects of control or asclla MOs on transgene expression in our $-1016 \alpha 1$ T:GFP transgenic fish. Normally, $\mathrm{GFP}^{+}$Müller glia are visible at $2 \mathrm{dpi}$, and by $4 \mathrm{dpi}$, there are numerous $\mathrm{GFP}^{+}$Müller glia (Fig. 1, -1016 panel). It was readily apparent that asclla knockdown suppressed transgene expression in Müller glia after retinal injury at both 2 and 4 dpi ( 4 dpi shown in Fig. 6). Specificity was confirmed using a second MO (asclla $a^{5^{\prime} \text { UTR }}$ ), which gave similar results. We counted the number of lissamine ${ }^{+} /$ $\mathrm{GFP}^{+}$cells in the inner nuclear layer (INL) and ONL by analyzing confocal images and found that $13 \%$ of the control MO-treated cells expressed GFP at $2 \mathrm{dpi}$, whereas only $4 \%$ the asclla MO-treated cells expressed GFP (three retinas were counted for each group; $p=0.02$ ). 
At 4 dpi, $22 \%$ of the control MO-treated cells expressed GFP, whereas only $2 \%$ of the asclla and asclla ${ }^{5^{\prime} U T R}$ MO-treated cells expressed GFP, respectively (three retinas were counted for each group; $p=0.03$ for the asclla $\mathrm{MO}, p=0.04$ for the asclla $\mathrm{a}^{5^{\prime} \mathrm{UTR}}$ $\mathrm{MO})$. These quantitative data confirm our qualitative observations and suggest asclla is required for $\alpha 1 T$ :GFP transgene expression in vivo.

Because asclla knockdown prevented the $\alpha 1 T$ transgene from being induced in response to injury, we wondered whether endogenous gene induction was also blocked. For these experiments, we used our $-1016 \alpha 1$ : GFP transgenic fish so we could monitor transgene GFP expression by fluorescence microscopy and endogenous $\alpha 1 T$ expression by in situ hybridization in control and asclla MO-treated retinas at $4 \mathrm{dpi}$. Although the control MO had no effect on transgene expression or endogenous $\alpha 1 T$ induction (Fig. $7 a-c$ ), asclla knockdown almost completely abolished transgene and endogenous $\alpha 1 T$ expression in the morpholino-treated portion of the injured retina (Fig. $7 d-f$ ).

We also examined whether asclla knockdown could suppress the increased expression of pax 6 that we observed in cycling progenitors located in the INL and ONL at 4 dpi (supplemental Table 1, available at www.jneurosci.org as supplemental material) (Fausett and Goldman, 2006). Because pax 6 is constitutively expressed by amacrine cells in the uninjured retina at a position in the INL that is closest to the inner plexiform layer (Fig. 7j), we compared ectopic pax6 expression in the INL and ONL of control and asclla MO-treated/injured retinas by ISH (Fig. $7 g-i$ ). Interestingly, asclla MO-treated retinas exhibited a dramatic reduction in ectopic pax6 expression (Fig. $7 j-l$ ) compared with control MO-treated retinas (Fig. $7 g-i$ ). The observation that pax6 expression persisted in amacrine cells of the asclla MOtreated/injured retina (Fig. 7i) suggests that these cells control pax6 expression in an asclla-independent manner and demonstrate the asclla MOs are not causing a general decrease in gene expression.

Ascl1a is required to convert Müller glia into cycling population of retinal progenitors

The early induction of asclla after retinal injury and its requirement for $\alpha 1 T$ expression suggests asclla induction may be necessary for retina regeneration to occur. One of the hallmarks of retina regeneration is the production of a cycling population of retinal progenitors derived from Müller glia. To determine whether asclla induction is necessary for the generation of this
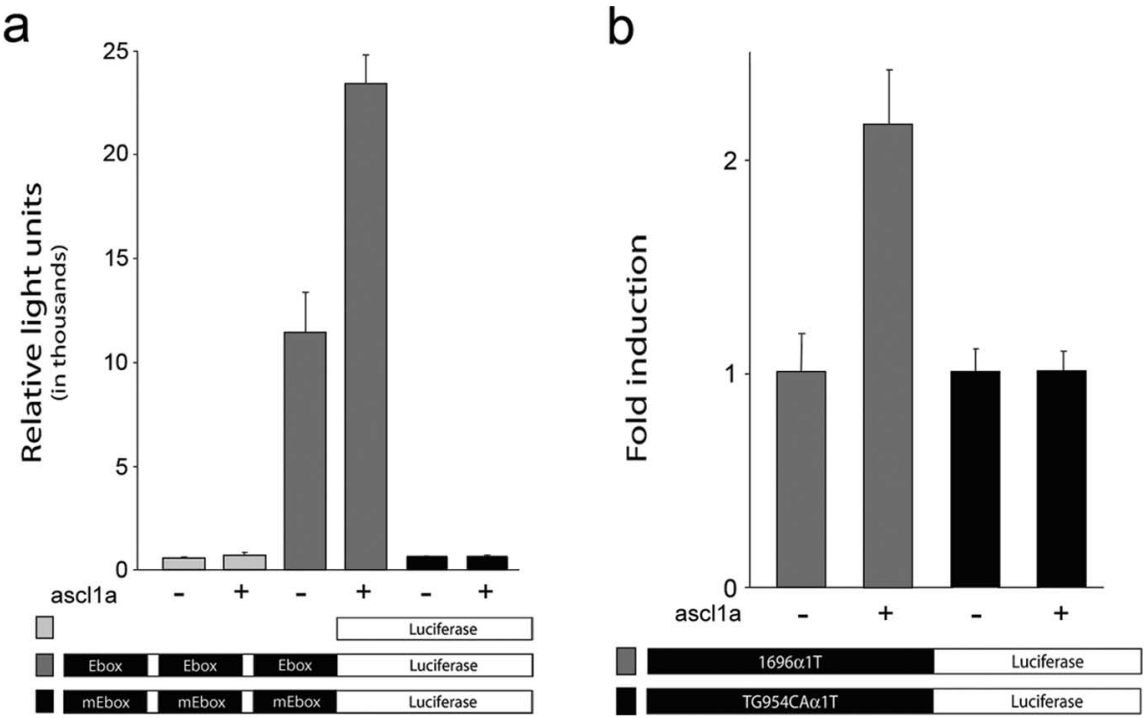

Figure 5. Ascl1a regulates the $\alpha 1 T$ promoter through the $-954 \mathrm{E}-\mathrm{box} . \boldsymbol{a}$, Luciferase reporter vectors with either a minimal $\beta$-globin promoter alone (light gray bars) or three copies of the $-954 \mathrm{E}$-box (dark gray bars) or the mutant E-box from probe $4-4$ (see Fig. 2b) (black bars) were transfected in combination with ascl1a into HEK293T cells. Ascl1a transactivates the reporter when a functional E-box is present but not when the E-box is mutated. $\boldsymbol{b}$, Full-length $\alpha 1 T$ constructs harboring either the wild-type (-1696) or mutantE-box promoters were transfected into HEK293T cells with or without ascl1a. Ascl1a induces reporter expression when the E-box is intact, but not when the E-box is mutated. Error bars indicate SEM for three replicates.
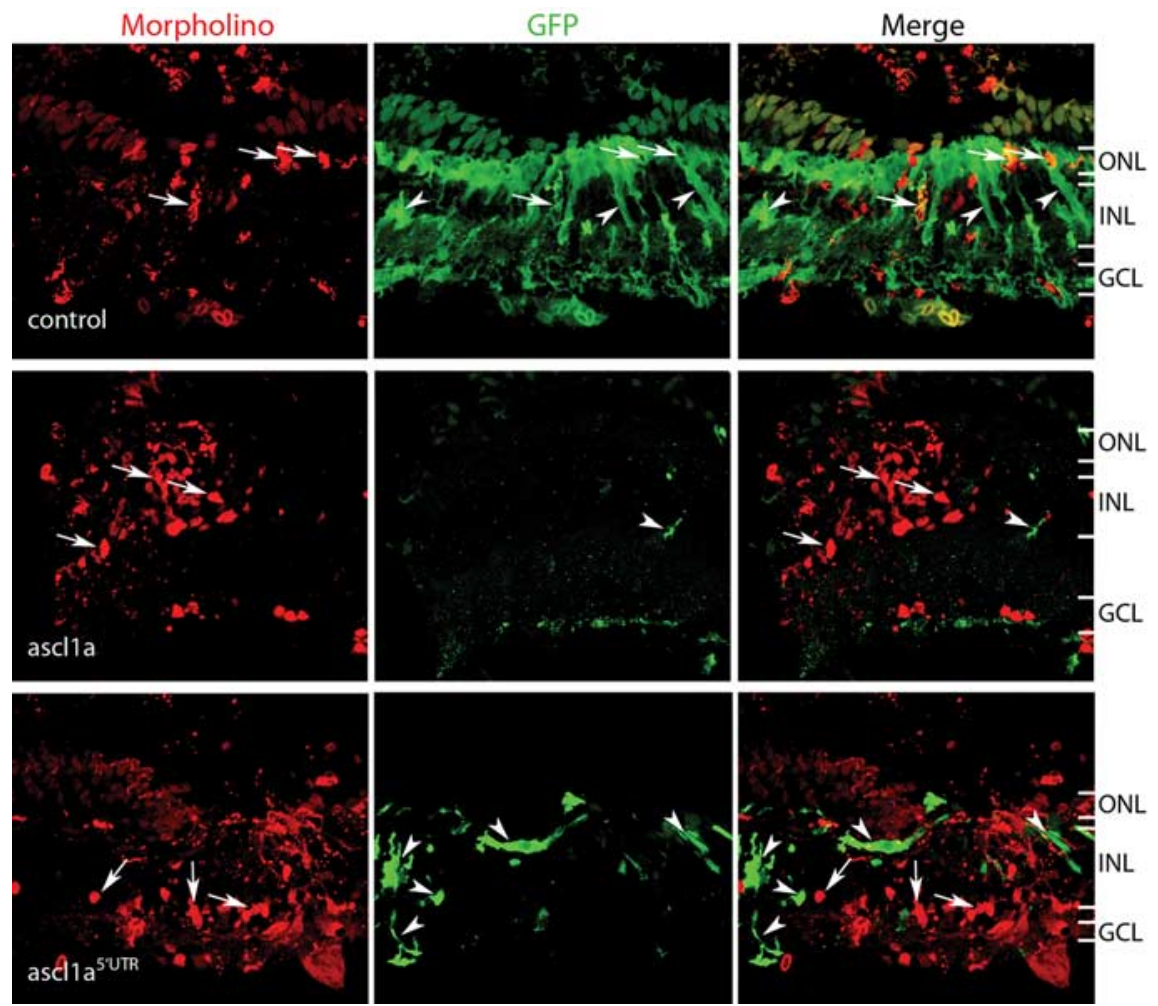

Figure 6. Ascl1a is required for transgene expression in vivo. - 1016 transgenic fish received retinal injuries and morpholino treatment on day 0 . Eyes were harvested on day 4. $\boldsymbol{a}$, Control morpholino treatment has no effect on transgene expression in Müller glia (arrows), and there are many GFP ${ }^{+}$Müller glia (arrowheads). Morpholinos targeting ascl1a (ascl1a and ascl1 $a^{{ }^{\prime} U^{\prime} T R}$ panels) prevent transgene expression in treated cells (arrows) and cause reduced transgene expression in general, although Müller glia that did not receive ascl1a morpholinos are able to express GFP (arrowheads). GCL, Ganglion cell layer.

cycling progenitor pool, we delivered asclla-targeted or control MOs to Müller glia in the injured retina. Because the vast majority of BrdU-labeled cells in the injured retina are derived from Müller glia (Fausett and Goldman, 2006), $\mathrm{MO}^{+} / \mathrm{BrdU}^{+}$cells 

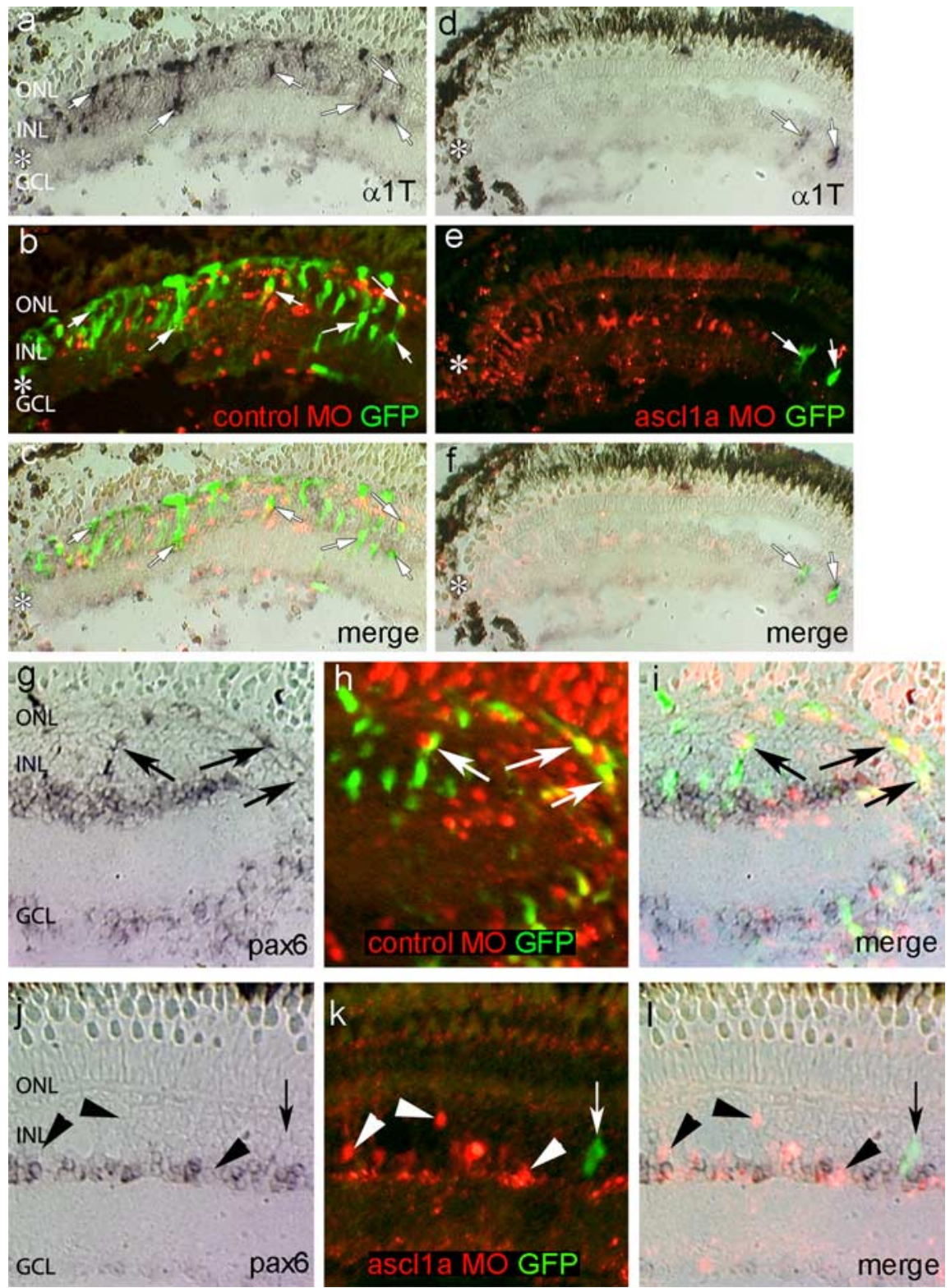

Figure 7. Ascl1a knockdown prevents induction of endogenous $\alpha 1 T$ and pax6. Retinas from -1016 transgenic fish were injured and electroporated with lissamine-labeled MOs on day 0 and harvested on day $4 . \boldsymbol{a}-\mathbf{f}, \alpha 1 T$ expression detected by ISH is shown as a dark brown deposit $(\boldsymbol{a}, \boldsymbol{d})$, native GFP expression is shown in green $(\boldsymbol{b}, \boldsymbol{e})$, and lissamine-labeled M0 is shown in red $(\boldsymbol{b}$, e). The injury site is marked by an asterisk. $\boldsymbol{a}-\boldsymbol{c}$, Control M0 treatment does not affect endogenous or transgene $\alpha 1 T$ induction (arrows). $\boldsymbol{d}-\mathbf{f}$, Ascl1a knockdown prevents endogenous and transgene $\alpha 1 T$ induction. Note the lack of $\alpha 1 T$ and GFP expression between the asterisk and the arrows, where the retina is treated with $\mathrm{M} 0$. Where the retina did not receive $\mathrm{M0}, \alpha 1 T$ and GFP are expressed (arrows). $\boldsymbol{g}-\mathbf{I}$, Pax6 expression detected by ISH is shown as a blue/purple deposit, native GFP expression is shown in green, and $\mathrm{M} 0$ is shown in red. $\boldsymbol{g}-\boldsymbol{i}$, Control $\mathrm{M} 0$ treatment does not affect pax6 induction at 4 dpi. Arrows indicate $p a x 6^{+} / \mathrm{MO}^{+} /$ $\mathrm{GFP}^{+}$Müller glia.j-I, Ascl1a M0 treatment prevents pax6 induction. Arrowheads indicate $\mathrm{MO}^{+} / \mathrm{pax}^{-}{ }^{-}$cells. Rare GFP ${ }^{+}$cells are sometimes present but were not treated with M0 (arrow).

represent injury-induced retinal progenitors. We therefore injured and simultaneously electroporated -1016:GFP transgenic retinas with lissamine-labeled control or asclla MOs and marked dividing cells by housing the fish in BrdU-treated water from 36 to $60 \mathrm{~h}$, when Müller glia are dividing. We harvested the retinas at $10 \mathrm{dpi}$ and quantified the number of lissamine ${ }^{+} / \mathrm{BrdU}^{+}$cells in the INL by analyzing confocal images of retinal sections (Fig. 8). We found $22 \%$ of the control MO-treated cells were labeled with BrdU, whereas two different asclla-targeting morpholinos resulted in only 5\% (5'UTR MO) and 3.5\% (ATG spanning MO) of the cells being labeled with BrdU (three retinas were counted for each group; $p<$ $0.05)$. Similar results were obtained when we assayed for the earliest proliferating cells by injuring and electroporating the retina on day 0 and housing the fish in BrdU-treated water from $24-48 \mathrm{~h}$ and immediately harvesting retinas to assay $\mathrm{MO}^{+} / \mathrm{BrdU}^{+}$cells (supplemental Fig. 5, available at www.jneurosci.org as supplemental material). Although the presence of asclla MO treated cells at 10 dpi suggests these MOs do not cause cell death, we examined whether any MO-treated cells were caspase $^{+}$at 2 or $4 \mathrm{dpi}$ and did not detect $\mathrm{MO}^{+} /$caspase $^{+}$cells, further suggesting asclla MOs do not cause cell death (data not shown). Together, these results suggest asclla knockdown prevents Müller glia from entering the cell cycle after injury.

\section{Discussion}

Zebrafish provide an ideal system for exploring the mechanisms underlying nervous system regeneration because of their robust regenerative response to injury and their amenability to experimental manipulation. We generated transgenic zebrafish models for studying regeneration in the zebrafish nervous system, and these models have further facilitated our ability to study the regenerative process (Goldman and Ding, 2000; Goldman et al., 2001; Senut et al., 2004; Fausett and Goldman, 2006). In particular, we used the $\alpha 1 T$ promoter driving EGFP expression as a reporter of the regenerative response. Transgenic fish harboring wild-type or mutant $\alpha 1 T$ promoters led us to a critical E-box that is necessary for transgene induction in retinal progenitors after injury. Investigation of the proteins that may activate the $\alpha 1 T$ promoter via this E-box led us to asclla, which proved to be a critical regulator of not only $\alpha 1 T$ promoter activity but also the regenerative response itself. Asclla was recently reported to be induced within $2 \mathrm{dpi}$, the earliest time point examined (Yurco and Cameron, 2007). Our data show ascl1a is one of the earliest gene inductions in the injured retina (first detectable by 4 hpi), preceding $\alpha 1$, olig2, pax 6 , notch, and delta by at least $18 \mathrm{~h}$. Based on their temporal expression pattern, most of these latter genes may contribute to maintaining a population of cycling progenitors, cell migration, and cell type specification. Delta-notch signaling has long been known to regulate cell fate decisions in Drosophila (Bray, 1998), and in zebrafish, notch signaling regulates neuronal cell type specification in the spinal cord (Shin et al., 2007). Pax6 has also been shown to play a role in specifying certain retinal cell types, because its removal at optic cup stages reduces the formation of most retinal neurons (Marquardt et al., 2001). $\alpha 1 \mathrm{~T}$ ex- 
pression may contribute to microtubules that are involved in cell division, cell migration, and cell shape changes.

\section{Ascl1 a regulates $\alpha 1 T$ transgene} expression in proliferating Müller glia We identified a critical E-box (CATGTG) at position -954 within the $\alpha 1 T$ promoter that is necessary for transgene expression in proliferating Müller glia after retinal injury. Although we demonstrated that this E-box mediates asclla-dependent promoter induction, we were unable to show a direct interaction between asclla and this E-box in gel electrophoretic mobility shift assays. This may suggest the conditions we used are not appropriate for binding, or that asclla has an indirect effect on $\alpha 1 T$ promoter activity. Although we cannot distinguish between these possibilities, we note that the critical E-box in the $\alpha 1 T$ promoter deviates from the consensus mouse achaete-scute complex-like 1 (Ascl1) binding site (CAGCTG) (Hu et al., 2004). In addition, $\alpha 1 T$ activation in the injured retina lags behind asclla induction by at least $14 \mathrm{~h}$ suggesting asclla may induce expression of other basic helix-loop-helix proteins or must cooperate with later induced proteins to bind the -954 E-box of the $\alpha 1 T$ promoter. Nonetheless, knockdown of asclla in the injured retina dramatically suppressed $\alpha 1 T$ :GFP transgene and endogenous $\alpha 1 \mathrm{~T}$ expression. By using two different MOs targeting different asclla sequences, we ensure specificity of these results. Therefore, we conclude that asclla regulates $\alpha 1 T$ expression in proliferating Müller glia.

\section{Asclla activates quiescent Müller glia to become multipotent progenitors}

Müller glia are quiescent cells that respond to retinal injury by generating multipotent retinal progenitors. $\alpha 1 T$ transgene expression, cell cycle re-entry, and expression of other injuryinduced genes are all indications that multipotent progenitors have been formed. What causes these glial cells to become actively dividing retinal progenitors? Although the signaling mechanisms are still unknown, the results presented here suggest asclla expression is necessary for converting quiescent Müller glia into actively proliferating progenitors. This conclusion is based on MO-mediated knockdown of asclla in adult Müller glia at the time of retinal injury, and the observation that asclla is induced within $4 \mathrm{hpi}$, which is $18 \mathrm{~h}$ before the beginning of Müller glia proliferation (Fausett and Goldman, 2006).

This proposed role for asclla in the regenerating retina is very different from its role in the developing retina, where ascll appears to collaborate with other bHLH and homeodomain transcription factors to initiate cell cycle exit and specify retinal neuronal subtype (Akagi et al., 2004; Wang and Harris, 2005; Guillemot, 2007). However, in other parts of the nervous system such as the medial ganglionic eminence of the telencephalon and olfactory epithelium (Cau et al., 1997; Casarosa et al., 1999), ascl1 does contribute to the generation of neural precursors, where it seems to play a role in specifying stem cells to commit to a par- ticular type of transiently proliferating neural progenitor (Torii et al., 1999). We suspect asclla expression in zebrafish Müller glia behaves similarly contributing to its transition from a quiescent cell to an actively dividing retinal progenitor. Although the mechanism by which asclla accomplishes this is not known, it is possible that the molecular environment and/or asclla expression level of Müller glia is such that asclla-dependent dedifferentiation and cellular proliferation are favored over cell-cycle exit and retinal cell type differentiation. Asclla likely specifies Müller glia to become injury-induced progenitors by collaborating with other transcription factors to initiate the expression of target genes such as $\alpha 1 T$ and cell cycle regulators (Kassen et al., 2007). Interestingly, the cell cycle regulator cyclin D1 is induced in the injured retina (Kassen et al., 2007) and harbors an E-box (CATGTG) in its promoter that matches the $\alpha 1 T$ asclla-dependent E-box in sequence and position (data not shown).

Might asclla also contribute to retinal cell type specification? This is an interesting question, because asclla is normally thought to contribute to cell cycle exit and cellular differentiation, and asclla expression rises to its maximal level at $\sim 48 \mathrm{hpi}$ (supplemental Table 1, available at www.jneurosci.org as supplemental material) and persists up to at least $7 \mathrm{dpi}$ (Yurco and Cameron, 2007). However, this question is difficult to address experimentally, because it would require us to knockdown asclla expression in the regenerating retina at a time when retinal progenitors are actively dividing (at $\sim 4 \mathrm{dpi}$ ). Because $\mathrm{MO}$-mediated gene knockdown is most effective in the vicinity where it is injected, this would require one to introduce the MO into the injured retina in the same place where the initial needle poke injury occurred, which is very difficult. Therefore, this question may be 
better addressed by using intense light to injure the retina, which results in a more uniform injury response (Kassen et al., 2007).

The rapid induction of asclla in Müller glia after retinal injury suggests it may be regulated by transcription factors already present in Müller glia. One such candidate is stat3, which is expressed in quiescent Müller glia and may be activated by injuryinduced extracellular cues (Kassen et al., 2007). Interestingly, FGF can cause Müller glia to re-enter the cell cycle in the absence of injury (Fischer et al., 2002), and asclla appears to act downstream of FGF signaling in developing zebrafish pituitary gland (Herzog et al., 2004). Another potential regulator is the wnt signaling pathway. In rats, adding wnt3a to retinal explant cultures increases Müller glia proliferation (Osakada et al., 2007). Wnt signaling is mediated by nuclear localization of $\beta$-catenin, which activates genes harboring $\mathrm{T}$ cell transcription factor binding sites. Whether wnt $3 \mathrm{a} / \beta$-catenin mediates its effect via asclla is not known. Future studies will begin to address the role these signal transduction cascades play in regeneration of the zebrafish retina.

\section{References}

Akagi T, Inoue T, Miyoshi G, Bessho Y, Takahashi M, Lee JE, Guillemot F, Kageyama R (2004) Requirement of multiple basic helix-loop-helix genes for retinal neuronal subtype specification. J Biol Chem 279:28492-28498.

Barthel LK, Raymond PA (2000) In situ hybridization studies of retinal neurons. Methods Enzymol 316:579-590.

Bernardos RL, Barthel LK, Meyers JR, Raymond PA (2007) Late-stage neuronal progenitors in the retina are radial Müller glia that function as retinal stem cells. J Neurosci 27:7028-7040.

Bray S (1998) Notch signalling in Drosophila: three ways to use a pathway. Semin Cell Develop Biol 9:591-597.

Cameron DA, Gentile KL, Middleton FA, Yurco P (2005) Gene expression profiles of intact and regenerating zebrafish retina. Mol Vis 11:775-791.

Casarosa S, Fode C, Guillemot F (1999) Mash1 regulates neurogenesis in the ventral telencephalon. Development 126:525-534.

Cau E, Wilson SW (2003) Ashla and neurogenin1 function downstream of floating head to regulate epiphysial neurogenesis. Development 130:2455-2466.

Cau E, Gradwohl G, Fode C, Guillemot F (1997) Mash1 activates a cascade of bHLH regulators in olfactory neuron progenitors. Development 124:1611-1621.

Fausett BV, Goldman D (2006) A role for alphal tubulin-expressing Müller glia in regeneration of the injured zebrafish retina. J Neurosci 26:6303-6313.

Fimbel SM, Montgomery JE, Burket CT, Hyde DR (2007) Regeneration of inner retinal neurons after intravitreal injection of ouabain in zebrafish. J Neurosci 27:1712-1724.

Fischer AJ, Reh TA (2001) Müller glia are a potential source of neural regeneration in the postnatal chicken retina. Nat Neurosci 4:247-252.

Fischer AJ, McGuire CR, Dierks BD, Reh TA (2002) Insulin and fibroblast growth factor 2 activate a neurogenic program in Müller glia of the chicken retina. J Neurosci 22:9387-9398.

Goldman D, Ding J (2000) Different regulatory elements are necessary for alpha1 tubulin induction during CNS development and regeneration. NeuroReport 11:3859-3863.

Goldman D, Hankin M, Li Z, Dai X, Ding J (2001) Transgenic zebrafish for studying nervous system development and regeneration. Transgenic Res 10:21-33.
Guillemot F (2007) Spatial and temporal specification of neural fates by transcription factor codes. Development 134:3771-3780.

Herzog W, Sonntag C, Walderish B, Odenthal J, Maischein HM, Hammerschmidt M (2004) Genetic analysis of adenohypophysis formation in zebrafish. Mol Endocrinol 18:1185-1195.

Hieber V, Dai X, Foreman M, Goldman D (1998) Induction of alpha1tubulin gene expression during development and regeneration of the fish central nervous system. J Neurobiol 37:429-440.

Hitchcock PF, Macdonald RE, VanDeRyt JT, Wilson SW (1996) Antibodies against Pax6 immunostain amacrine and ganglion cells and neuronal progenitors, but not rod precursors, in the normal and regenerating retina of the goldfish. J Neurobiol 29:399-413.

Hu Y, Wang T, Stormo GD, Gordon JI (2004) RNA interference of the achaete-scute homolog 1 in mouse prostate neuroendocrine cells reveals its gene targets and DNA binding sites. Proc Natl Acad Sci USA 101:5559-5564.

Jasoni CL, Reh TA (1996) Temporal and spatial pattern of MASH 1 expression in the developing rat retina demonstrates progenitor cell heterogeneity. J Comp Neurol 369:319-327.

Kassen SC, Ramanan V, Montgomery JE, Burket C, Liu CG, Vihtelic TS, Hyde DR (2007) Time course analysis of gene expression during lightinduced photoreceptor cell death and regeneration in albino zebrafish. Develop Neurobiol 67:1009-1031.

Marquardt T, Ashery-Padan R, Andrejewski N, Scardigli R, Guillemot F, Gruss P (2001) Pax6 is required for multipotent state of retinal progenitor cells. Cell 105:43-55.

Mensinger AF, Powers MK (2007) Visual function in regenerating teleost retina following surgical lesion. Vis Neurosci 24:1-9.

Nordeen SK (1998) Luciferase reporter gene vectors for analysis of promoters and enhancers. BioTechniques 6:454-458.

Ooto S, Akagi T, Kageyama R, Akita J, Mandai M, Honda Y, Takahashi M (2004) Potential for neural regeneration after neurotoxic injury in the adult mammalian retina. Proc Natl Acad Sci USA 101:13654-13659.

Osakada F, Ooto S, Akagi T, Mandai M, Akaike A, Takahashi M (2007) Wnt signaling promotes regeneration in the retina of adult mammals. J Neurosci 27:4210-4219.

Senut M, Gulati-Leekha A, Goldman D (2004) An element in the $\alpha 1$ tubulin promoter is necessary for retinal expression during optic nerve regeneration but not after eye injury in the adult zebrafish. J Neurosci 24:7663-7673.

Shin J, Poling J, Park H, Appel B (2007) Notch signaling regulates neural precursor allocation and binary neuronal fate decisions in zebrafish. Development 134:1911-1920.

Torii M, Matsuzaki F, Osumi N, Kaibuchi K, Nakamura S, Casarosa S, Guillemot F, Nakafuku M (1999) Transcription factors Mash-1 and Prox-1 delineate early steps in differentiation of neural stem cells in the developing central nervous system. Development 126:443-456.

Vihtelic TS, Hyde DR (2000) Light-induced rod and cone cell death and regeneration in the adult albino zebrafish (Danio rerio) retina. J Neurobiol 44:289-307.

Wang JC-C, Harris WA (2005) The role of combinational coding by homeodomain and bHLH transcription factors in retinal cell fate specification. Dev Biol 285:101-115.

Yun K, Fischman S, Johnson J, Hrabe de Angelis M, Weinmaster G, Rubenstein JL (2002) Modulation of the notch signaling by Mash1 and Dlx1/2 regulates sequential specification and differentiation of progenitor cell types in the subcortical telencephalon. Development 129:5029-5040.

Yurco P, Cameron DA (2007) Cellular correlates of proneural and notchdelta gene expression in the regenerating zebrafish retina. Vis Neurosci 24:437-443. 